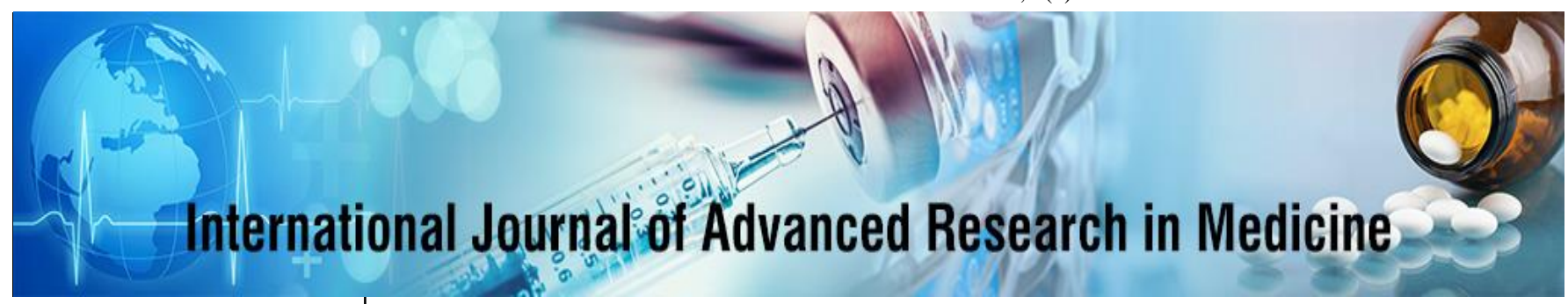

E-ISSN: 2706-9575

P-ISSN: 2706-9567

www.medicinepaper.net

IJARM 2021; 3(2): 104-106

Received: 10-03-2021

Accepted: 29-06-2021

Dr. Madhumati Varma

Assistant Professor,

Department of General

Medicine, Jaipur National

University Institute for

Sciences and Research Centre

JNUIMSRC, Rajasthan, India

Dr. Ashok Dutt Mathur Professor, Department of General Medicine, Jaipur National University Institute for Sciences and Research Centre JNUIMSRC, Rajasthan, India
Corresponding Author: Dr. Madhumati Varma Assistant Professor, Department of General Medicine, Jaipur National University Institute for Sciences and Research Centre JNUIMSRC, Rajasthan, India

\section{To evaluate the relationship diabetes mellitus and thyroid dysfunction (Thyrobetes) in JNU Hospital, Jaipur}

\author{
Dr. Madhumati Varma and Dr. Ashok Dutt Mathur
}

DOI: $\underline{\text { https://doi.org/10.22271/27069567.2021.v3.i2b.225 }}$

\begin{abstract}
Diabetes and thyroid endocrine diseases which interlinked, function of one gland can affect other so it is really necessary to evaluate how they influence each other when not functioning well.

There were done study on diabetic and thyroid patient, data collected basis of demographic, duration of diseases, analysis which important like Glucose, TSH etc., data analyze in excel software, found diabetes mellitus type 2 amongst more fallowed by hypothyroidism and" Thyrobetes" about $10.7 \%$.

Introduction: Diabetes and thyroid endocrine diseases which interlinked, function of one gland can affect other so it is really necessary to evaluate how they influence each other when not functioning well.

Objective of study: To define interrelation of factors responsible for interlink between diabetes and thyroid disease development.

Method and Methodology: This study evaluates duration diseases of thyroid and diabetes with basic analysis like glucose, TSH Hbalc, lipid profile etc. and data analyses in excel software done, found diabetes mellitus type 2 amongst more number and percentage of patients fallowed by hypothyroidism and" Thyrobetes" about $10.7 \%$ and DM type $252 \%$, hypothyroidism $49 \%$. P was significant $<0.02$.

Conclusion: There should be very necessary to make policies to evaluate Diabetic patients with periodically thyroid function test, and thyroid patient evaluate glucose level vice versa so on time controlled both diseases.
\end{abstract}

Keywords: Thyrobetes, diabetes, thyroid, hypo and hyper thyroid, glucose, TSH

\section{Introduction}

As India registered women unhealthy then men. As per the GOQii diabetes has increased from 7.1 to 12 percent this year. "The incidence of diabetes is highest among older adults at 23.81 percent and seniors at 36.82 percent. As per the report, 13.5 percent of Indians have cholesterol issues. This past year, thyroid issues have increased from 6.8 to 10.7 percent. The "Thyrobetes" is illustrating relationship between thyroid diseases and diabetes mellitus. There is environment of risk to develop diabetes and thyroid dysfunction. The association of hypothyroidism and worsening of diabetes started since $1927^{[1]}$.

There is question: Thyroid diseases are at risk for development diabetes or diabetes at risk for development of thyroid disease? These diseases overlap each other might call mutual destructive relationship.

Hyperthyroidism: Glucose comes out from liver from break down glycogen which causes glucose intolerance and insulin resistance. Higher T3 level has high risk to develop Type 2 Diabetes.

Hypothyroidism: Reduces glycemic index (GI) glucose absorption and increase glucose in blood with decrease output glucose from liver. Body produces more glucose by gluconeogenesis because of not getting diet and brain craves energy. Muscle using less glucose, insulin resistance and low HDL. Increase risk of diabetes when TSH level above 2.5 and beta cell function decrease and insulin resistance increases. The gene GLUT4, transporter of glucose controlled by T3 can elevated transport both ways passive and insulin mediated. 


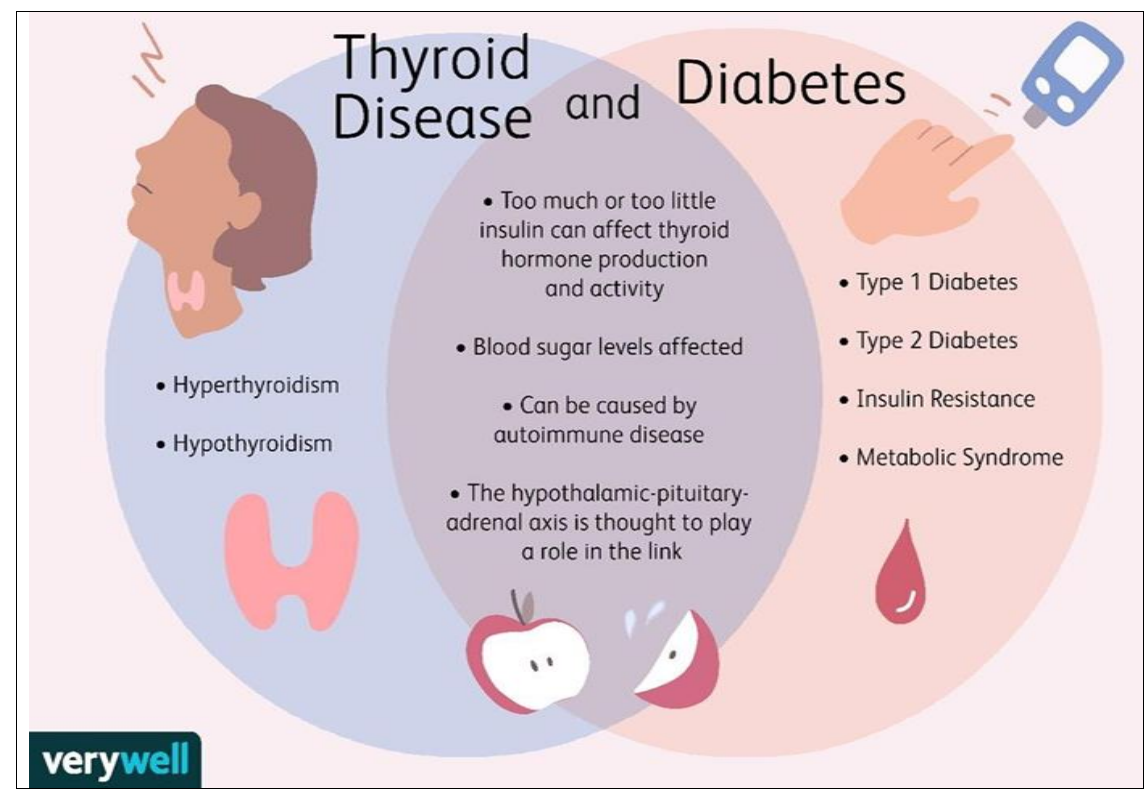

\section{Objective of the study}

On completion of study will be able to find out:

a. To define interrelation of factors responsible for interlink between diabetes and thyroid disease development.

b. To estimate thyroid hormone TSH, free T3, free T4, lipid profile, blood glucose fasting and PP, HbAlc, S. Insulin, ant-TPO antibodies, USG neck for thyroid some patients.

c. To describe each factor correlation between developing diabetes and thyroid diseases.

d. To explain preventive measures to prevent both diseases.

\section{Research problem of the study}

There are patients increasing of diabetes mellitus, hypothyroidism and other condition of thyroid dysfunction but difficult to say which disease occurs first. Study is going to find out overlapping risk factor between diabetes and thyroid dysfunction.

\section{Review of literature}

Type 2 diabetes mellitus and thyroid dysfunction (TD) are two major public health endocrine problems in world. The status of iodine and thyroid with diabetic patients less studied. Few studies available which done on this relationship are:

TSH rise responsible for development of diabetic neuropathy and diabetic nephropathy and one of four people of diabetes are suffering with thyroid diseases ${ }^{[1]}$. Routine biochemical screening of geriatric patients done, in which increased prevalence of subclinical hypothyroidism ${ }^{[2]}$. Prevalence of hypothyroidism was found high in type $2 \mathrm{DM}$ patients above 45 years and more so if their BMI is over $25 \%{ }^{[3]}$. Another study done showed female suffered more thyroid dysfunction compare to man, fasting glucose and lipid profile deranged, TSH significantly high among diabetic patients ${ }^{[4]}$. Type 2 diabetes found subclinical hypothyroidism and risk of neuropathy and cardiac events as thyroid hormone control insulin secretion: hypothyroidism decrease insulin secretion by glucose level and hyperthyroidism causes insulin resistance ${ }^{[5]}$. Female found central obesity, DM nephropathy, above normal
$\mathrm{HbAlc}$, and duration of DM were risk factors of thyroid dysfunction in type 2 DM patients in this study ${ }^{[6]}$.

\section{Identification of research gap}

There is very no study to evaluated factors those interlink, overlap and interdependent, initiation for development diabetes and thyroid dysfunction.

\section{Framework and Methods for proposed research project Materials and Methods}

The study will be carried out in the research unit in JNU Hospital, medicine department, Jaipur. The study included 477 DM patients of any age group, and 50-age matched healthy control. The questionnaires were used to the record the findings on general history, physical examination thyroid hormone TSH, free T3, free T4, lipid profile, blood glucose fasting and $\mathrm{PP}, \mathrm{HbA1c}$, S. Insulin, ant-TPO antibodies, USG neck for thyroid some patients.

\section{Inclusion criteria}

- Sample size-477 patients.

- No limit age group.

- Newly or follow up cases of diabetic mellitus and thyroid disease.

\section{Exclusion criteria}

- Patients having acute illness which affect thyroid gland activity.

- The patients having drugs which influence the thyroid hormone status.

- The patients who had fever.

Data collection and tool for statistical analysis

Instruments used for analyzing the Samples taken at JNU hospital.

\section{Statistical analysis}

Statistical analysis was performed by using excel software, correlation was used and if the $\mathrm{P}^{*}$ value is $<0.005$ then it is considered to be statistically significant. Data organized in excel and make descriptive analysis of date as well as graphs, finding as follows: 
Table 1: Shows diseases and their descriptive analysis with duration of diseases

\begin{tabular}{|c|c|c|c|c|c|c|c|c|}
\hline & DM Type 2 & Duration & Prediabetic & Duration & Hypothyroid & Duration & Hyperthyroidism & Duration \\
\hline Count & 249 & & 14 & 11 & 235 & & 26 & \\
\hline Countbla & 227 & & 462 & & 240 & & 450 & \\
\hline Average & 1 & 5.576923 & & & 1 & 3.3434389 & & 0.98 \\
\hline Median & 1 & 4 & & & 1 & 2 & & \\
\hline St Dev & & 5.611851 & & & & 3.9011328 & & 1.09702 \\
\hline Percentage & & 52 & & 2.9 & & 49 & & 5.4 \\
\hline
\end{tabular}

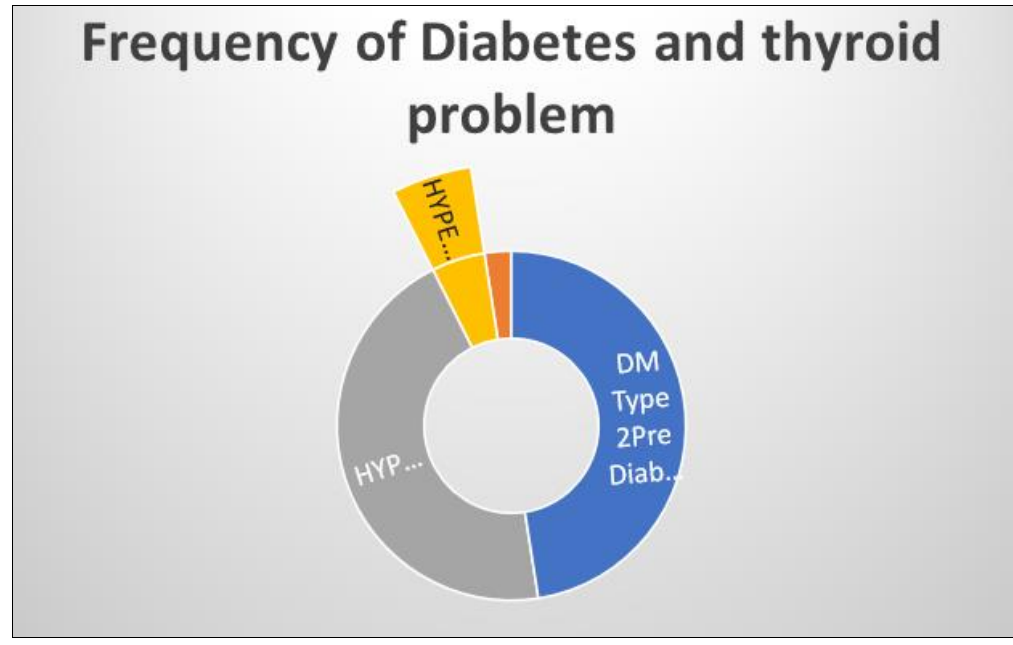

Fig 1: Show prevalence of type of diabetes and thyroid problem

Percentage: DM type 2-52\%, Pre-Diabetic-2.9\%, Hypothyroidism 49\%, Hyperthyroidism $5.4 \%$, Those both hypothyroidism/hyper with DM type $2-10.71 \%$.

In which common Diabetic and thyroid both "Thyrobetes" were about $10.71 \%$.

\section{Discussion of result}

There is found that amongst study papulation predominant DM type 2 then hypothyroidism and those overlapping thyroid and diabetes was $10.71 \%$. There were significant correlation $P<0.01$. As we know those has hypothyroidism insulin resistant and slow development diabetes if nit controlled well. Also, if diabetic uncontrolled high TSH can developed due to lipid deranged, low production of Free T3.

\section{Innovativeness in the proposed research project}

There will be evaluated factors those interlink, overlap and interdependent, initiation for development diabetes and thyroid dysfunction.

\section{Relevance of the proposed research project}

Health policy to those Diabetic should regularly evaluate for thyroid function and those thyroid diseases evaluate for diabetic investigation regularly.

\section{References}

1. Ghosh S, Pramanik S, Mukhopadhyay P, Bhattacharjee $\mathrm{R}$, Mukherjee B, Mondal S et al. Thyroid status in patients with Type 2 diabetes attending a Tertiary Care Hospital in Eastern India. Indian Journal of Endocrinology and Metabolism 2018;22(1):112. Doi: 10.4103/ijem.ijem_572_17

2. Feely J, Isles TE. Screening for thyroid dysfunction in diabetics. BMJ 1979;2(6202):1439-1439. Doi: 10.1136/bmj.2.6202.1439-b.

3. Laloo Demitrost $\mathrm{S}$. Thyroid dysfunction in type 2 diabetes mellitus: A retrospective study. [online]
PubMed Central (PMC) 2020. Available at: https://www.ncbi.nlm.nih.gov/pmc/articles/PMC36030 66/ [Accessed 13 Feb. 2020].

4. Sonali Chaturvedi, Suryakant Nagtilak, Pawan Parashar, Amit Rastogi, Akash Gupta. Thyroid dysfunction and its relation with Type 2 Diabetes Mellitus in Meerut International Journal of Scientific Research 2016, 5(7).

5. Prevalence and predictors of thyroid dysfunction among patients with type 2 diabetes mellitus attending a tertiary care hospital in an urban area of Bhubaneswar, Odisha.

6. Ogbonna SU, Ezeani IU. Risk Factors of Thyroid Dysfunction in Patients with Type 2 Diabetes Mellitus. Frontiers in Endocrinology 2019, 10. Doi: $10.3389 /$ fendo.2019.00440 